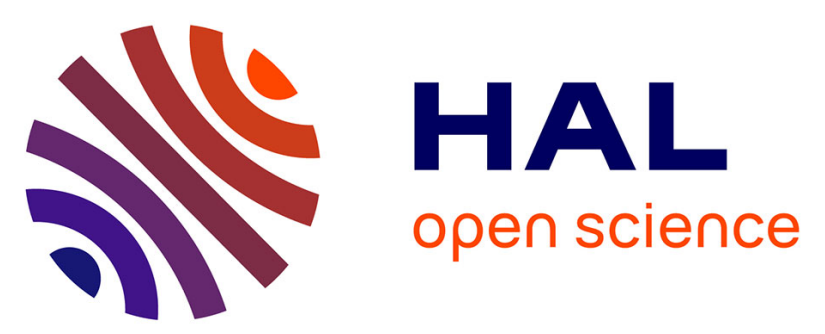

\title{
A reduced-order model of detuned cyclic dynamical systems with geometric modifications using a basis of cyclic modes
}

M. Mbaye, Christian Soize, J.-P. Ousty

\section{- To cite this version:}

M. Mbaye, Christian Soize, J.-P. Ousty. A reduced-order model of detuned cyclic dynamical systems with geometric modifications using a basis of cyclic modes. Journal of Engineering for Gas Turbines and Power-Transactions of the Asme, 2010, 132 (11), pp.Article Number 112502. 10.1115/1.4000805 . hal-00684307

\section{HAL Id: hal-00684307 \\ https://hal.science/hal-00684307}

Submitted on 1 Apr 2012

HAL is a multi-disciplinary open access archive for the deposit and dissemination of scientific research documents, whether they are published or not. The documents may come from teaching and research institutions in France or abroad, or from public or private research centers.
L'archive ouverte pluridisciplinaire HAL, est destinée au dépôt et à la diffusion de documents scientifiques de niveau recherche, publiés ou non, émanant des établissements d'enseignement et de recherche français ou étrangers, des laboratoires publics ou privés. 


\title{
A reduced-order model of detuned cyclic dynamical systems with geometric modifications using a basis of cyclic modes
}

\author{
Moustapha Mbaye ${ }^{1,2}$, Christian Soize ${ }^{1 *}$, Jean-Philippe Ousty ${ }^{2}$ \\ ${ }^{1}$ Université Paris-Est, Laboratoire de Modélisation et Simulation Multi Echelle \\ MSME FRE3160 CNRS, 5 bd Descartes, 77454 Marne-la-Vallée, France \\ 2 Turbomeca - Safran Group, 64511 Bordes, France \\ moustapha.mbaye@univ-paris-est.fr \\ christian.soize@univ-paris-est.fr \\ jean-philippe.ousty@turbomeca.fr
}

A new reduction method for vibration analysis of intentionally mistuned bladed disks is presented. The method is built for solving the dynamic problem of cyclic structures with geometric modifications. It is based on the use of the cyclic modes of the different sectors which can be obtained from a usual cyclic symmetry modal analysis. Hence the projection basis is constituted as well as, on the whole bladed disk, each sector matrix is reduced by its own modes. The method is validated numerically on a real bladed disk model, by comparing free and forced responses of a full model finite element analysis to those of a reduced-order model using the new reduction method.

\section{Introduction}

In the context of turbomachinery design, small variations in the blade characteristics of cyclic structures due to manufacturing tolerances affect the structural cyclic symmetry creating mistuning which increases the forced response amplitudes (e.g. see [1-3]). However, it is possible (e.g. see [4-8]) to intentionally mistune the mistuned system in order to reduce the forced response amplification. Intentionally mistuning the system is called detuning. The main technical solutions to introduce detuning are based on modifying blade material properties, the interface between the blades and the disk, or the blades' shapes by introducing several types of blades with different geometries corresponding to geometric modifications of the nominal blades. In the present paper, it is assumed that detuning is performed by modifying blade shapes and the disk is not detuned.

Vibration analyses of cyclic structures are usually performed using their cyclic symmetry and formulated for one sector from which the dynamic of the whole structure is obtained (e.g. see [9-12]). This is no longer the case for mistuned and/or detuned structures which need a full structure

*Adress all correspondance to this author. formulation. To reduce numerical computational costs while solving the detuning problem on finite element meshes of realistic bladed disks, many reduced-order methods have been introduced (see [6,13-20]). In general, reduced-order models are obtained by substructuring a bladed disk into disk and blades components (see e.g. [13,14]), as this allows an easy implementation of blade mistuning or detuning. However, a different approach [15] called SNM has been proposed by Yang and Griffin, in which the tuned system cyclic modes are used without substructuring to generate a reduced-order model. This technique is very efficient in the case of cyclic structures with blade material properties modifications but can be not efficient for the case of blade geometric modifications for the following reasons. It is well known that the tuned modes constitute a basis of the admissible space of the displacements for the mistuned bladed disk. Nevertheless, such a basis is generally not really efficient with respect to the convergence speed and a large number of tuned modes are required for such a situation. It should be noted that if convergence is slow, then the reduced-order model is not sufficiently small and so would not be efficient and effective to implement the probabilistic model of uncertainties and above all to perform a robust design optimization. In another hand, it is well recognized today that, if the use of the tuned modes for constructing the reduced-order model is efficient for blade material properties modifications, it is not always the case for blade geometric modifications (see for instance $[18,21])$. In addition, the blade geometric modifications induce a problem related to incompatibility representations between the tuned modes calculated with the mesh of the tuned bladed disk and the structural matrices of the geometrically modified bladed disk calculated with another mesh. In these conditions, the nominal and the geometrically modified meshes are incompatible (see Appendix A). Consequently, these incompatible finite element meshes induce a difficulty for constructing the projection of the geometri- 
cally modified mass and stiffness matrices using the tuned bladed disk sector cyclic modes. In such a situation, intrusive developments must be performed in commercial softwares to take into account this incompatibility of representations related to different meshes. A simple model derived from SNM and known as the Fundamental Mistuning Model (FMM) that reduces the set of nominal modes to a single modal family $[16,17]$ had also been introduced. Nevertheless, its application field is limited to a modal family with nearly equal frequency. An extension of the FMM for the case in which all modes of the family do not share the same frequency, known as Asymptotic Mistuning model (AMM) has also been introduced in $[19,20]$. This method is a perturbation method in which the small parameters are the small frequency corrections induced by mistuning, the small damping of the tuned modes or the first aerodynamic correction of the purely structural vibration characteristics. Then large geometric detuning can be difficult to solve with this method. To solve the problem of geometric detuning, without using substructuring, a method named Static Mode Compensation (SMC) has been proposed in [18], and used in [22], in which the mistuned system is represented by the full tuned system and by virtual mistuning components. But this method needs a convergence acceleration to be performed, which leeds to a large amount of calculation.

In this paper, we propose a method inspired of the SNM method but allowing the case of geometric detuning to be treated with efficiency with respect to the speed of convergence and with respect to the incompatibility of the representations induced by the use of different finite element meshes corresponding to the nominal blade and to the geometrically modified blades. Thus, we do not want to have a substructuring method or to use static modes for convergence acceleration. Then, it is assumed that a commercial software (black box) is used to compute the cyclic modes and mass and stiffness matrices of the different bladed disk sector types in independent calculations. In this particular context, we propose here a new method which uses the cyclic modes of the different bladed disk sectors and which consist on reducing each sector mass and stiffness matrices by its own modes. Linear constraints are applied on common boundaries between sectors to make the displacement field admissible on the entire bladed disk. An application is done on a realistic bladed disk model by comparing its dynamic characteristics using this reduction method to those obtained on a full model.

\section{Theory}

\subsection{Dynamic equation}

Let us consider the finite element model $\Omega$ of a detuned structure with $N$ blades. Detuning results from geometric modifications of some blades. In the frequency band $\mathbb{F}$ defined by $\mathbb{F}=\left[\omega_{\min }, \omega_{\max }\right], 0<\omega_{\min }<\omega_{\max }$ the dynamic equation of the detuned bladed disk can be written

$$
\left(-\omega^{2}[M]+j \omega[D]+[K]\right) \mathbf{u}(\omega)=\mathbf{f}(\omega),
$$

where the vector $\mathbf{u}$ stands for the union of all sectors (with free interfaces) displacements for the entire detuned bladed disk and not the displacement vector of the entire detuned bladed disk. This vector contains redundant degrees-offreedom corresponding to the common interfaces degrees-offreedom of adjacent sectors (a blade + a part of the disk) due to the fact we consider each sector with its inner and boundaries degrees-of-freedom. f denotes the vector of external structural forces and fluid-structure coupling forces, matrices $[M],[D],[K]$ represent real mass, damping and stiffness matrices and $j^{2}=-1 . n_{\text {dof }}$ is the size of vectors $\mathbf{u}$.

Linear constraint equations must be added to Eq.(1). The vector $\mathbf{u}(\omega)$ is written as

$$
\mathbf{u}(\omega)=\left(\mathbf{u}_{\ell}(\omega), \mathbf{u}_{c}(\omega)\right)
$$

in which $\mathbf{u}_{c}(\omega)$ is the vector of constrained degrees-offreedom and where $\mathbf{u}_{\ell}(\omega)$ is the vector of the free degreesof-freedom. The constraints equation can the be written as

$$
\mathbf{u}_{c}(\omega)=[B] \mathbf{u}_{\ell}(\omega)
$$

It can then be deduced the constraints equation for the entire detuned bladed disk,

$$
\mathbf{u}(\omega)=[\mathbb{B}] \mathbf{u}_{\ell}(\omega)
$$

Introducing the dynamic stiffness matrix

$$
[E(\omega)]=-\omega^{2}[M]+j \omega[D]+[K],
$$

then the dynamic equation (1) integrating the constraints equation becomes

$$
[\mathbb{B}]^{T}[E(\omega)][\mathbb{B}] \mathbf{u}_{\ell}(\omega)=[\mathbb{B}]^{T} \mathbf{f}(\omega)
$$

The detuned bladed disk is made of $\mathrm{N}$ sectors compatible on their coupling interfaces. Consequently, the dynamic stiffness matrix is formed by $N \times N$ sub-matrices, each one having $\bar{n} \times \bar{n}$ components and displacement and forces vectors are formed by $N$ sub-vectors.

$$
\begin{gathered}
{[E]=\left(\begin{array}{ccc}
{[E]^{0}} & \cdots & {[0]} \\
\vdots & \ddots & \vdots \\
{[0]} & \cdots & {[E]^{N-1}}
\end{array}\right)} \\
\mathbf{u}=\left(\begin{array}{c}
\mathbf{u}^{0} \\
\vdots \\
\mathbf{u}^{N-1}
\end{array}\right), \quad \mathbf{f}=\left(\begin{array}{c}
\mathbf{f}^{0} \\
\vdots \\
\mathbf{f}^{N-1}
\end{array}\right)
\end{gathered}
$$


with the vector $\mathbf{u}^{p}$ containing the displacements of the $\bar{n}$ degrees-of-freedom associated with sector $p$. Note that matrix $[E]$ is a bloc diagonal matrix. Then, the mass and stiffness matrices take the form

$$
\begin{gathered}
{[M]=\left(\begin{array}{ccc}
{[M]^{0}} & \cdots & {[0]} \\
\vdots & \ddots & \vdots \\
{[0]} & \cdots & {[M]^{N-1}}
\end{array}\right),} \\
{[K]=\left(\begin{array}{ccc}
{[K]^{0}} & \cdots & {[0]} \\
\vdots & \ddots & \vdots \\
{[0]} & \cdots & {[K]^{N-1}}
\end{array}\right),}
\end{gathered}
$$

where the $\bar{n} \times \bar{n}$ sector mass and stiffness matrices $[M]$ and $[K]$ are symmetric matrices and

$$
\begin{aligned}
& {[M]^{p}=\left(\begin{array}{ccc}
{\left[M_{l l}\right]} & {\left[M_{l i}\right]} & {[0]} \\
{\left[M_{l i}\right]^{T}} & {\left[M_{i i}\right]} & {\left[M_{i r}\right]} \\
{[0]} & {\left[M_{i r}\right]^{T}} & {\left[M_{r r}\right]}
\end{array}\right),} \\
& {[K]^{p}=\left(\begin{array}{ccc}
{\left[K_{l l}\right]} & {\left[K_{l i}\right]} & {[0]} \\
{\left[K_{l i}\right]^{T}} & {\left[K_{i i}\right]} & {\left[K_{i r}\right]} \\
{[0]} & {\left[K_{i r}\right]^{T}} & {\left[K_{r r}\right]}
\end{array}\right)}
\end{aligned}
$$

where subscripts $i, l$ and $r$ are related to the inner, the left side coupling interface and the right side coupling interface degrees-of-freedom. At this step, the dynamic system matrices and the displacement vector are expressed in local blade coordinates system associated with each sector $\Omega_{p}, \quad \forall p \in\{0, N-1\}$.

\subsection{Reduced-order model \\ 2.2.1 Projection basis}

Let us consider $t$ different blade types for a bladed disk with $N$ blades, $t \leq N$. In the following discussion, we consider $N$ blade types which can be different, knowing that if $t<N$ there is at least one repetition of a blade type. So, each sector $\Omega_{p}$ of the detuned bladed disk is associated with a blade type. For each blade type associated with a given $\Omega_{p}$, a tuned bladed disk is considered with this blade type in cyclic symmetry conditions. For each tuned bladed disk associated with $\Omega_{p}$, the cyclic modes are computed. Let $\widehat{\psi}_{1}^{\Omega_{p}}, \ldots, \widehat{\psi}_{m}^{\Omega_{p}}$ be the $m$ cyclic modes in the global coordinates system of the tuned bladed disk whose blade type is associated with the blade type of sector $\Omega_{p}$ in the detuned bladed disk. For the projection basis, the continuity of the displacement vector is ensured between two adjacent sectors which have the same blade type, but is not ensured between two adjacent sectors with different blade types. In addition, when the cyclic modes of two different sector types are computed by independent calculations, a phase shift can appear between them. This phenomenon will be explained more in subsection 2.2.2. So we need to reinstate the displacement vector continuity and put in phase the modes related to different sector types over the entire bladed disk. It should be noted that if all blades are identical, then the projection basis integrates the displacement vector continuity between adjacent sectors and the phase coherence which makes the constraint Eq.(2) automatically satisfied. In the global coordinates system, the projection basis is written as

$$
[\widehat{\Psi}]=\left(\begin{array}{ccc}
\widehat{\psi}_{1}^{\Omega_{0}} & \cdots & \widehat{\psi}_{m}^{\Omega_{0}} \\
\vdots & \ddots & \vdots \\
\widehat{\psi}_{1}^{\Omega_{N-1}} & \cdots & \widehat{\psi}_{m}^{\Omega_{N-1}}
\end{array}\right)
$$

where $\widehat{\psi}_{\alpha}^{\Omega_{p}}$ is a real mode expressed in the global coordinates system, $m$ is the number of modes selected and $\Omega_{p}$ is a geometrical domain represented by the sector $p$ of the detuned bladed disk. The subscript $\alpha$ stands for a couple of index $(n, v)$ where $n$ is the number of nodal diameters of the mode and $v$ is the mode rank for this family of nodal diameters. So, $\widehat{\psi}_{\alpha}^{\Omega_{p}}$ is the mode number $\alpha$ associated with sector $p$ which has a blade of type $\Omega_{p}$. This real mode is obtained using the corresponding cyclic mode $\phi_{(n, v)}^{0, p}=\widetilde{\phi}_{(n, v)}^{0, p}+\underset{j \widetilde{\phi}_{(n, v)}^{0, p}}{(n+2)}$ in local coordinate system of sector $\Omega_{0}$ for the bladed disk of type $\Omega_{p}$ in cyclic symmetry conditions

$$
\widehat{\psi}_{(n, v)}^{\Omega_{p}}=\widetilde{\phi}_{(n, v)}^{0, p} \cos \left(\frac{2 n p \pi}{N}\right)-\widetilde{\widetilde{\phi}}_{(n, v)}^{0, p} \sin \left(\frac{2 n p \pi}{N}\right)
$$

\subsubsection{Phase correction of cyclic modes of different sec- tor types}

To built a basis for the complete detuned bladed disk with cyclic modes of the different sector types, we must write the continuity and phase conditions on interfaces between adjacent sectors with different blade types, as said previously. In fact, since cyclic modes of each type of sector are computed independently from the cyclic modes of the other sector types, a phase shift may appear between cyclic modes of different sector types. This phase shift is caracterized by a rotation of the nodal diameters of the cyclic mode on the disk. In fact, in tuned conditions, the location of the modes nodal diameters on the bladed disk is indeterminate as shown in Fig. 1. On this figure, the sub-figures on left and right represent twin orthogonal modes obtained for each computation. To ensure the phase coherence between the cyclic modes of the different sector types, we introduce a complex parameter called MSF (Modal Scale Factor) which is defined by

$$
\operatorname{MSF}(\mathbf{x}, \mathbf{y})=\frac{\{\mathbf{x}\}^{T}\{\overline{\mathbf{y}}\}}{\{\mathbf{x}\}^{T}\{\overline{\mathbf{x}}\}}
$$

where $\arg M S F(\mathbf{x}, \mathbf{y})$ represents the phase shift between the two complex vectors $\mathbf{x}$ and $\mathbf{y}$. In these conditions, each cyclic 


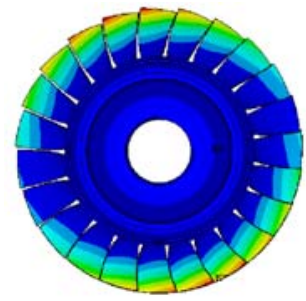

(a)

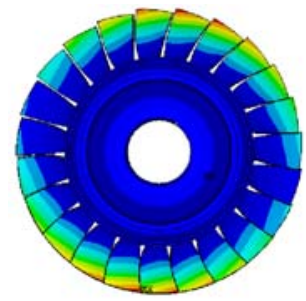

(c)

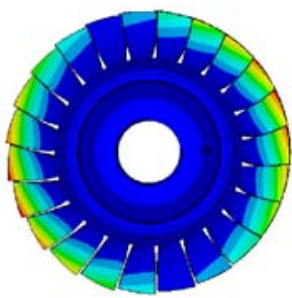

(b)

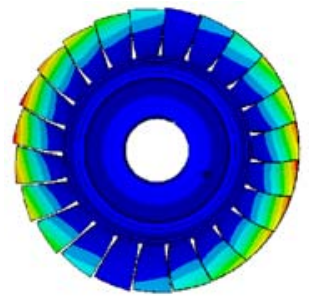

(d)

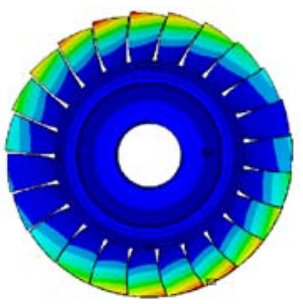

(a)

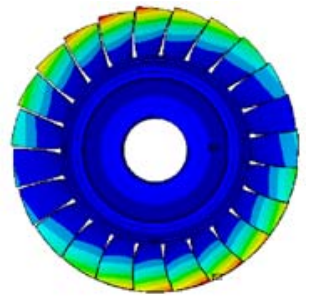

(c)

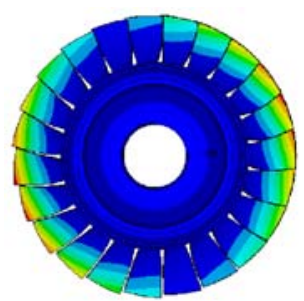

(b)

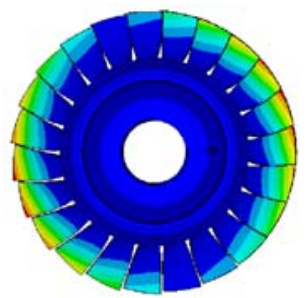

(d)
Fig. 1. Geometries of twin modes with one nodal diameter obtained by two independent computations : first computation $((a),(b))$ and second computation $((\mathrm{c}),(\mathrm{d}))$.

mode $\phi^{i n i}$ can be corrected in phase with the formula

$$
\phi=\frac{M S F\left(\phi^{n o m}, \phi^{i n i}\right)}{\left|M S F\left(\phi^{n o m}, \phi^{i n i}\right)\right|} \phi^{i n i}
$$

where $\phi^{i n i}$ a cyclic mode obtained by one of the independent computations, $\phi$ is a cyclic mode corresponding to $\phi^{i n i}$ and corrected in phase with $\phi^{n o m} . \phi^{n o m}$ is a cyclic mode obtained by one of the independent computations and taken as phase reference and $|z|$ represents the modulus of complex $z$. Thus, we can verify in Eq.(17) and Eq.(18) that the new mode $\phi$ is in phase with the nominal mode $\phi^{\text {nom }}$ because its MSF referred to the nominal mode is real, implying that its argument is null.

$$
\begin{aligned}
& \operatorname{MSF}\left(\phi^{n o m}, \phi\right)=\frac{\left\{\phi^{n o m}\right\}^{T}\{\bar{\phi}\}}{\left\{\phi^{n o m}\right\}^{T}\left\{\bar{\phi}^{n o m}\right\}} \\
& =\frac{\overline{M S F\left(\phi^{n o m}, \phi^{i n i}\right)}}{\left|M S F\left(\phi^{n o m}, \phi^{i n i}\right)\right|} \frac{\left\{\phi^{n o m}\right\}^{T}\left\{\overline{\phi^{i n i}}\right\}}{\left\{\phi^{n o m}\right\}^{T}\left\{\overline{\phi^{n o m}}\right\}} \\
& =\left|M S F\left(\phi^{\text {nom }}, \phi^{i n i}\right)\right| \in \mathbb{R}
\end{aligned}
$$

This implies that

$$
\arg \operatorname{MSF}\left(\phi^{\text {nom }}, \phi\right)=0
$$

Then after a phase correction of cyclic modes obtained by independent computations, Fig. 1 becomes Fig. 2, which exhibits phased cyclic mode shapes.

\subsubsection{Displacement continuity between adjacent sectors}

To solve the dynamic equation of motion of the geometrically detuned bladed disk, by using a projection basis
Fig. 2. Geometries of twin modes with one nodal diameter obtained by two independent computations after phase correction: first computation ((a),(b)) and second computation ((c),(d)).

made of different sector types cyclic modes, we must ensure an admissible displacement field on the coupling sector interfaces. This admissibility is defined by two conditions on interfaces: a compatibility of meshes which is naturally ensured because geometric modifications are only done on blades, and the displacement vector continuity between two adjacent sectors which can be ensured by linear constraints on the interface. In the method proposed, the displacements are taken equal on the coupling interface between two adjacent sectors. These constraints can be introduced by using a Lagrange multiplier field (see [23]) or by constraining the redundant degrees-of-freedom in each sector displacement vector. The latter formulation is made here. Let us consider Eq.(1) expressed in the global coordinates system and for which the constraints are not yet specified

$$
\left(-\omega^{2}[\widehat{M}]+j \omega[\widehat{D}]+[\widehat{K}]\right) \widehat{\mathbf{u}}(\omega)=\widehat{\mathbf{f}}(\omega)
$$

where $\widehat{\mathbf{u}}(\omega)=\left(\widehat{\mathbf{u}}_{\ell}(\omega), \widehat{\mathbf{u}}_{c}(\omega)\right), \widehat{\mathbf{u}}_{\ell}(\omega)$ is the displacement vector for free degrees-of-freedom and $\widehat{\mathbf{u}}_{c}(\omega)$ is the displacement vector for constrained degrees-of-freedom. The constraint relationship is written in the global coordinates system

$$
\widehat{\mathbf{u}}_{c}(\omega)=[\widehat{B}] \widehat{\mathbf{u}}_{\ell}(\omega)
$$

With this relationship, we can write

$$
\widehat{\mathbf{u}}(\omega)=[\widehat{\mathbb{B}}] \widehat{\mathbf{u}}_{\ell}(\omega) \quad .
$$

To reduce the detuned bladed disk model with a usefull basis, while respecting the specified constraints, we just have 
to project $\widehat{\mathbf{u}}(\omega)$ on a subspace which respects the constraints. This means that the basis vectors just have to respect these constraints. The modes of the basis $[\widehat{\Psi}]$ have been corrected in phase but are not continuous on degrees-of-freedom associated with interfaces between sectors. That is to say that they do not respect the constraints. Consequently, we are going to build a new projection basis $\left[\widehat{\Psi}^{\text {proj }}\right]$ from the modes of the basis $[\widehat{\Psi}]$ and which integrates the continuity constraints on sector interfaces. Let $\widehat{\psi}_{\alpha}^{\text {proj }}$ be a column of the new projection basis $\left[\widehat{\Psi}^{\text {proj }}\right]$. This vector is written $\widehat{\psi}_{\alpha}^{p r o j}=\left(\left(\widehat{\psi}_{\alpha}^{p r o j}\right)_{\ell},\left(\widehat{\psi}_{\alpha}^{p r o j}\right)_{c}\right)$. The constraint relationship is then written $\left(\widehat{\psi}_{\alpha}^{p r o j}\right)_{c}=[\widehat{B}]\left(\widehat{\psi}_{\alpha}^{\text {proj }}\right)_{\ell}$, which yields the equation

$$
\widehat{\psi}_{\alpha}^{p r o j}=[\widehat{\mathbb{B}}]\left(\widehat{\psi}_{\alpha}^{p r o j}\right)_{\ell}
$$

\subsubsection{System reduction}

The displacement vector can be projected on the built projection basis by

$$
\widehat{\mathbf{u}}(\omega)=\left[\widehat{\Psi}^{p r o j}\right] \mathbf{q}(\omega)
$$

where $\mathbf{q}=\left(\mathbf{q}_{1}, \ldots, \mathbf{q}_{m}\right)$ is the vector of generalized coordinates. Then the reduced problem can be written

$$
\left[\underline{\underline{E}}_{r e d}(\omega)\right] \underline{\mathbf{q}}(\omega)=\underline{\mathbf{g}}(\omega)
$$

where the reduced dynamic matrix $\left[\widehat{E}_{\text {red }}(\omega)\right]$ and the vector of generalized forces $\widehat{\mathbf{g}}$ are written as

$$
\begin{gathered}
{\left[\widehat{E}_{r e d}(\omega)\right]=\left[\widehat{\Psi}^{\text {proj }}\right]^{T}[\widehat{E}(\omega)]\left[\widehat{\Psi}^{\text {proj }}\right]} \\
\widehat{\mathbf{g}}(\omega)=\left[\widehat{\Psi}^{\text {proj }}\right]^{T} \widehat{\mathbf{f}}(\omega)
\end{gathered}
$$

Using the form of matrix $[E(\omega)]$ in Eq.(7) and the form of $\left[\widehat{\Psi}^{\text {proj }}\right]$ which can be deduce from Eq.(13), the reduced dynamic matrix $\left[\widehat{E}_{r e d}(\omega)\right]$ is fully populated and the terms of this matrix are written

$$
\left[\widehat{E}_{r e d}(\omega)\right]_{\beta, \alpha}=\sum_{p=0}^{N-1}\left(\left(\widehat{\psi}^{p r o j}\right)_{\beta}^{\Omega_{p}}\right)^{T}[\widehat{E}(\omega)]^{p p}\left(\left(\widehat{\psi}^{p r o j}\right)_{\alpha}^{\Omega_{p}}\right)
$$

\subsubsection{Particular case of a structural damping intro- duced by a modal damping ratio}

The way the reduced structural damping matrix is written by using the projection basis $\left[\widehat{\Psi}^{\text {proj }}\right]$ depends on the way structural damping is taken into account. In fact, when damping is taken into account by a fully populated matrix or a matrix expressed in function of the mass and the stiffness matrices $([\widehat{D}]=a[\widehat{M}]+b[\widehat{K}])$, the reduced structural damping matrix is fully populated with this projection basis. Although, if we consider a modal damping ratio, it is necessary to diagonalize the structural mass and stiffness reduced matrices to be able to write the reduced structural damping matrix in a diagonal form. To do so, we solve at first the homogenous eigenvalue problem without damping for the structural reduced system. Then we diagonalize the structural mass and stiffness matrices using the eigenvectors obtained. The homogenous eigenvalue problem to solve without damping for the structural reduced system is written: Find $(\lambda, \widehat{\mathbf{y}})$, with $\widehat{\mathbf{y}}$ so that

$$
\left(\left[\widehat{K}_{r e d}\right]-\lambda\left[\widehat{M}_{r e d}\right]\right) \widehat{\mathbf{y}}=0
$$

where $\lambda$ is an eigenvalue and $\widehat{\mathbf{y}}$ is an eigenvector. Since matrices $\left[\widehat{K}_{r e d}\right]$ and $\left[\widehat{M}_{\text {red }}\right]$ are real symmetric, their eigenvectors are real. Thus the eigenvectors obtained verify the orthogonality property

$$
<\left[\widehat{K}_{r e d}\right] \widehat{\mathbf{y}}^{\alpha}, \widehat{\mathbf{y}}^{\beta}>=\omega_{\alpha}^{2} \mu_{\alpha} \delta_{\alpha \beta}
$$

$$
<\left[\widehat{M}_{r e d}\right] \widehat{\mathbf{y}}^{\alpha}, \widehat{\mathbf{y}}^{\beta}>=\mu_{\alpha} \delta_{\alpha \beta}
$$

where $\mu_{\alpha}$ is the generalized mass associated with the mode $\alpha$ and $\omega_{\alpha}$ is the eigenfrequency associated with the mode $\alpha$. By choice, the reduced structural damping matrix can them be written in a diagonal form using a modal damping ratio

$$
<\left[\widehat{D}_{r e d}\right] \widehat{\mathbf{y}}^{\alpha}, \widehat{\mathbf{y}}^{\beta}>=2 \xi_{\alpha} \omega_{\alpha} \mu_{\alpha} \delta_{\alpha \beta}
$$

where $\xi_{\alpha}$ is the modal damping ratio associated with the mode $\alpha$. Note that the real modes $\widehat{\mathbf{u}}^{\alpha}$ of the detuned bladed disk in the global coordinates system are written as

$$
\widehat{\mathbf{u}}^{\alpha}=\left[\widehat{\Psi}^{\text {proj }}\right] \widehat{\mathbf{y}}^{\alpha}
$$

Let us introduce a new variable $\eta$ such that $\mathbf{q}(\omega)=[\widehat{\mathbf{y}}] \eta(\omega)$. Then the generalized problem defined by Eq.(24) becomes

$$
\left\{-\omega^{2}\left[\widehat{M}_{\text {diag }}\right]+i \omega\left[\widehat{D}_{\text {diag }}\right]+\left[\widehat{K}_{\text {diag }}\right]\right\} \eta(\omega)=[\widehat{\mathbf{y}}]^{T} \widehat{\mathbf{g}}_{\text {exc }}(\omega),
$$

where $\left[\widehat{M}_{\text {diag }}\right],\left[\widehat{K}_{\text {diag }}\right]$ and $\left[\widehat{D}_{\text {diag }}\right]$ are diagonal and are written as

$$
\begin{gathered}
{\left[\widehat{M}_{\text {diag }}\right]=[\widehat{\mathbf{y}}]^{T}\left[\widehat{M}_{\text {red }}\right][\widehat{\mathbf{y}}]=\left(\begin{array}{ccc}
\mu_{1} & \cdots & 0 \\
\vdots & \ddots & \vdots \\
0 & \cdots & \mu_{m}
\end{array}\right)} \\
{\left[\widehat{K}_{\text {diag }}\right]=[\widehat{\mathbf{y}}]^{T}\left[\widehat{K}_{\text {red }}\right][\widehat{\mathbf{y}}]=\left(\begin{array}{ccc}
\omega_{1}^{2} \mu_{1} & \cdots & 0 \\
\vdots & \ddots & \vdots \\
0 & \cdots & \omega_{m}^{2} \mu_{m}
\end{array}\right)}
\end{gathered}
$$




$$
\left[\widehat{D}_{\text {diag }}\right]=[\widehat{\mathbf{y}}]^{T}\left[\widehat{D}_{\text {red }}\right][\widehat{\mathbf{y}}]=\left(\begin{array}{ccc}
2 \xi_{1} \omega_{1} \mu_{1} & \cdots & 0 \\
\vdots & \ddots & \vdots \\
0 & \cdots & 2 \xi_{m} \omega_{m} \mu_{m}
\end{array}\right)
$$

\section{Validation of the reduction method with an industrial bladed disk}

The realistic test case considered here is an industrial bladed disk with 23 blades (see Fig. 3). The commercial software used to compute the cyclic modes and mass and stiffness matrices for the reduced-order model (ROM) inputs, and the forced response and resonant frequencies of the full model is ANSYS. Fig. 4 displays the eigenfrequencies

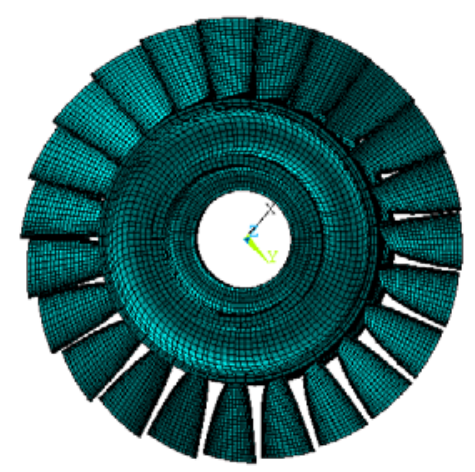

Fig. 3. Finite element model of the tuned bladed disk.

of the generalized eigenvalue problem associated with the tuned bladed disk as function of the circumferential wave number. To validate the method, we are going to approxi-

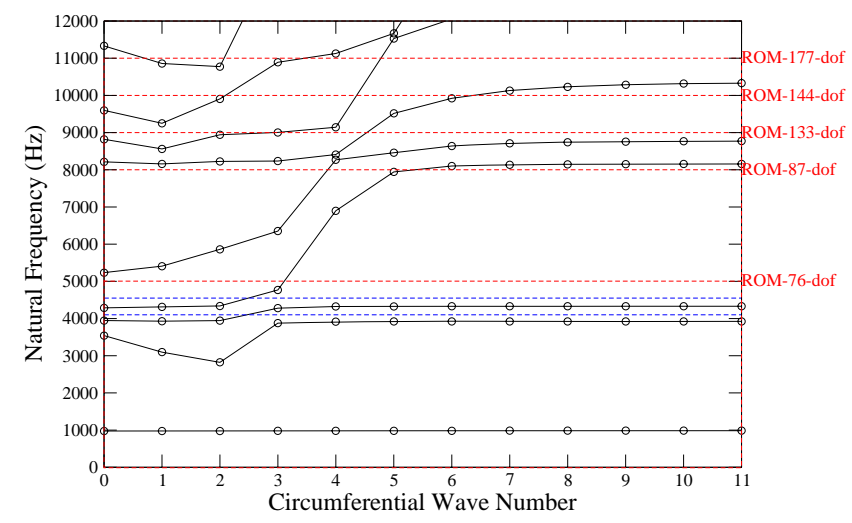

Fig. 4. Natural frequencies versus circumferential wave numbers of the tuned bladed disk.

mate the 76 first modes of the detuned bladed disk and to compute the forced response under two engine order excitation numbers. Note that the 76 first natural frequencies of the tuned bladed disk are above $5000 \mathrm{~Hz}$. For the detuned system, two other kinds of blades are created from the nominal one by shape modification of the blade upper part: a blade with increased thickness called "heavy blade" and a blade with decreased thickness called "light blade" (see Fig. 5). To quantify the level of mistuning, the three

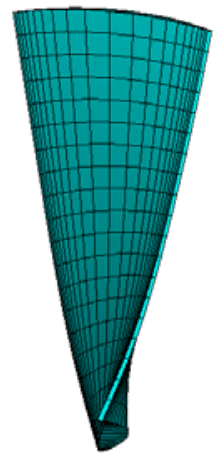

(a)

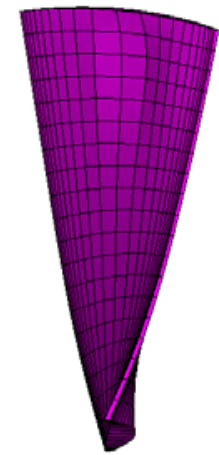

(b)

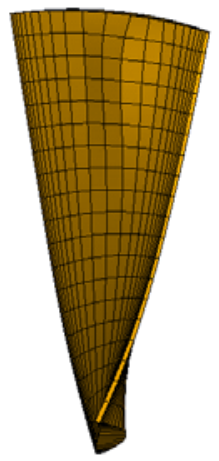

(c)
Fig. 5. Finite element models of blades: a reference blade (a), a light blade (b) and a heavy blade (c).

first natural frequencies of the different kinds of blades are computed in clamped blade alone configuration and shown in Table 1. These values show a large frequency deviation which can exceed $10 \%$ for the mode $2 \mathrm{~T}$. The bladed disk is

\begin{tabular}{|c|c|c|c|}
\hline & Nominal blade & Light blade & Heavy blade \\
\hline Mode 1F & 996.4 & 1054.3 & 947.9 \\
\hline Mode 2F & 4013.0 & 3937.5 & 4077.1 \\
\hline Mode 1T & 4382.6 & 4514.2 & 4321.9 \\
\hline Mode 2T & 8217.0 & 7616.6 & 9081.8 \\
\hline
\end{tabular}

Table 1. Natural clamped blades frequencies $(\mathrm{Hz})$ for the four first modes.

detuned by modifying arbitrarily two of its blades to make them have the shapes shown in Fig. 5. The test case we are going to study is shown in Fig. 6. Fig. 7 and Fig. 8 display the 76 first eigenfrequencies of the generalized eigenproblem associated with the detuned bladed disk and the corresponding frequency estimation errors for a ROM containing 76 dof. In this test, the eigenfrequencies prediction errors obtained for all approximated detuned modes are below $0.35 \%$, which demonstrates a sufficient accuracy in capturing the eigenfrequencies of the detuned bladed disk. Note that the maximum error levels are obtained for eigenfrequencies associated with modes for which the vibrational energy is mainly located on the disk, and that the eigenfrequencies associated with modes for which the vibrational energy is 
(c)

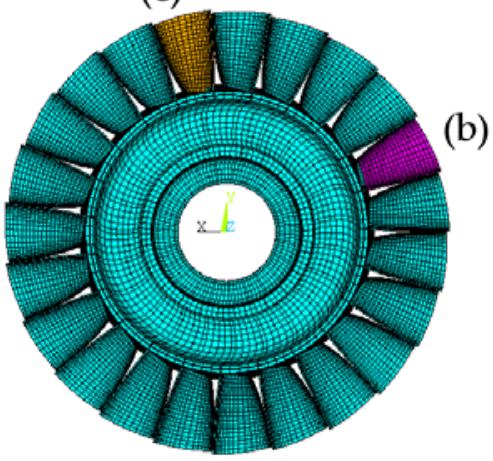

Fig. 6. Complete intentionally detuned bladed disk with arbitrary geometric modification of two blades.

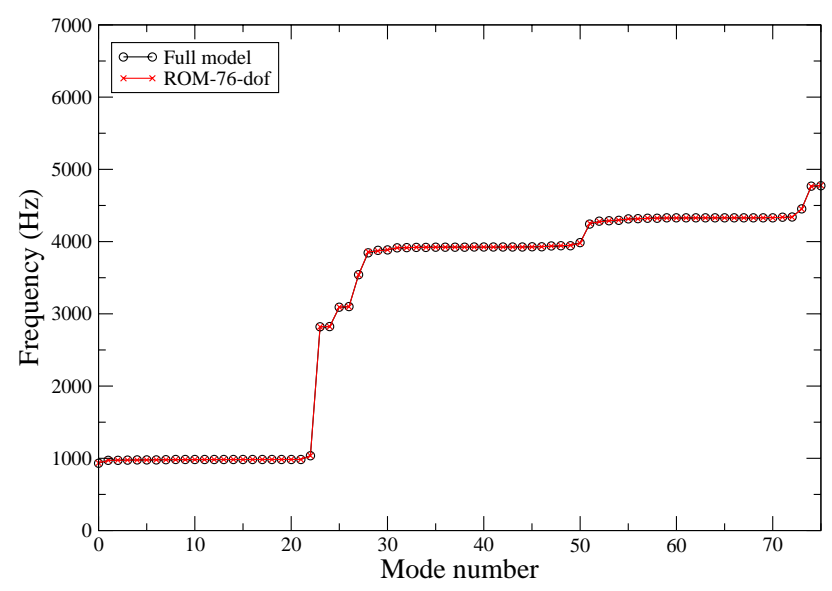

Fig. 7. Comparison of the 76 first detuned eigenfrequencies between the full model and several ROM sizes.

mainly located on the blades are precisely obtained, with an error level below $0.01 \%$. The latter case eigenfrequencies can be easily identified as the clustered modes near frequencies $1000 \mathrm{~Hz}, 4000 \mathrm{~Hz}$ and $4400 \mathrm{~Hz}$ in Fig. 7. For the forced response consideration, all blades responses under engine orders excitation 5 and 9 are computed in the frequency band $\mathbb{F}=[4150,4550] \mathrm{Hz}$ and displayed in Fig. 9 and Fig. 10. The ROM used for these calculations is a ROM with 92 dof. The structural damping is introduced with a damping ratio value of 0.003 . On these figures, both the full model and the ROM exhibit a clustered response of 21 blades and 2 isolated responses which are the modified blades responses. Moreover, the dynamic behavior of the full bladed disk model is quite similar to the ROM one. In Fig. 11 a comparison of the maximum forced response of the detuned bladed disk obtained with the full model and the ROM is presented. This comparison shows very good results since the curves are quite identical. The results obtained above demonstrate a sufficient accuracy of the proposed ROM in capturing the detuned bladed disk behavior, in both free and forced response con-

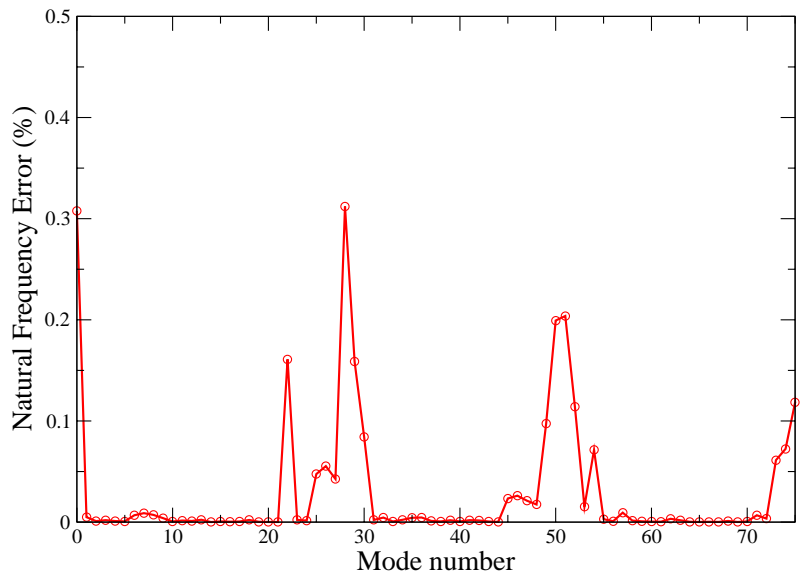

Fig. 8. Comparison of the 76 first detuned eigenfrequencies prediction errors between the full model and several ROM sizes.
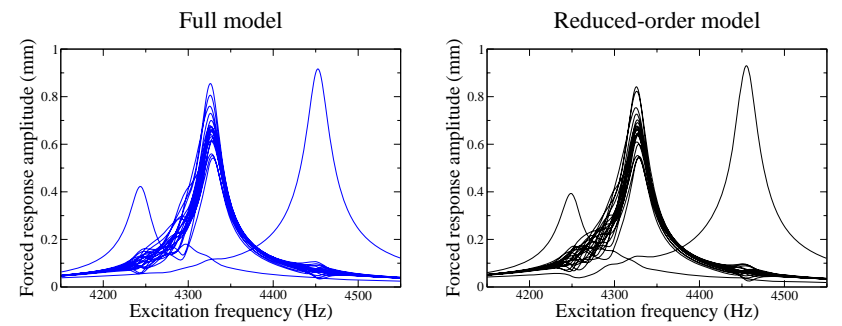

Fig. 9. Forced response of the 23 blades to an engine order excitation 5 in the frequency band $\mathbb{F}$ : Full model (left) and ROM (right).
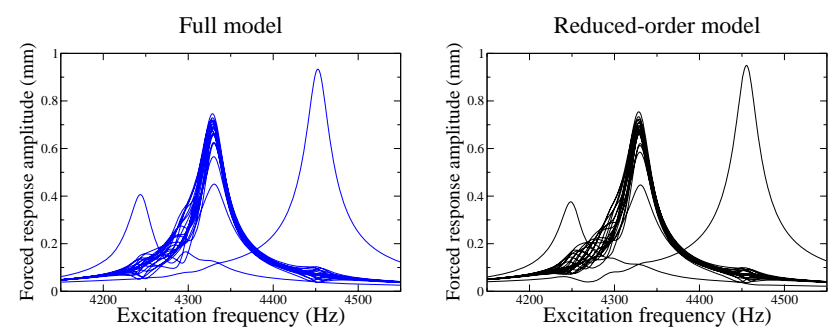

Fig. 10. Forced response of the 23 blades to an engine order excitation 9 in the frequency band $\mathbb{F}$ : Full model (left) and ROM (right).

figurations.

\section{Conclusion}

In this paper a new reduction method for the dynamic problem of cyclic structures with geometric modifications of blades, based on the use of the cyclic modes of the different sectors is presented. The projection basis is constituted as well as on the whole bladed disk, each sector matrix is reduced by its own modes and linear constraints are applied on common boundaries between sectors to make the displacement field admissible. This method is applied on an industrial bladed disk to show its efficiency. The proposed method allows very compact reduced-order models to be obtained and accurately capture the mistuned system dynamics. 

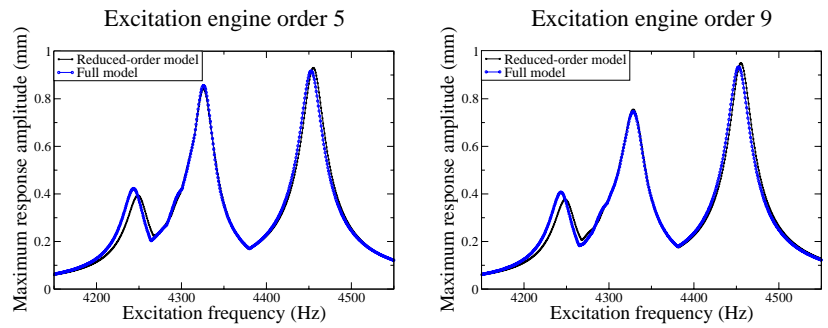

Fig. 11. Comparison of the maximum forced response of the bladed disk obtained with the full and the reduced-order models in the frequency band $\mathbb{F}$ : Excitation engine order 5 (left) and excitation engine order 9 (right).

\section{Appendix A}

This appendix is concerned with showing mesh incompatibility which can occur while projecting matrices of a component mesh on modes of a different geometric component mesh. A test case is made by modelling two plates. These plates have 70 elements : 1 in the thickness direction (z), 7 in the width direction (y) and 10 in the length direction (x) (cf. Fig. 12). Each element has 8 nodes. The only difference between these plates is that the modified plate has 4 nodes which have been moved in the thickness direction to get a local lower thickness, compared to the nominal plate. For the boundary conditions, the plates are clamped on the section $\mathrm{x}=0$. Fig. 13 shows the 4 first modes of the two plates. Let $\left[M^{\text {nom }}\right],\left[K^{\text {nom }}\right]$ and $\left[\phi^{\text {nom }}\right]$ be the mass matrix,
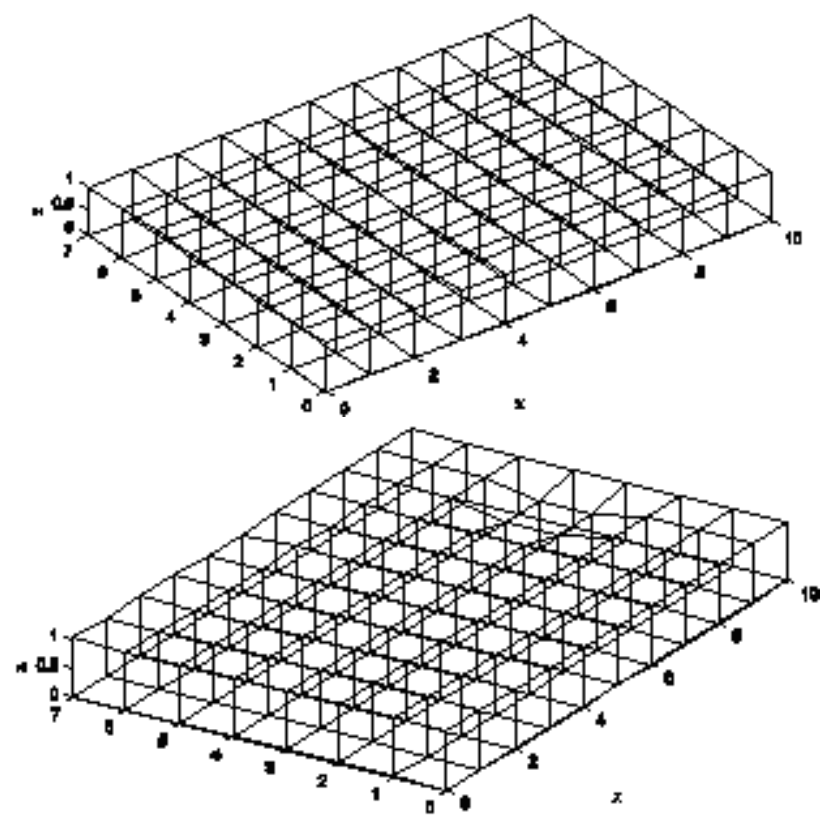

Fig. 12. Nominal plate (up) and geometrically modified blade (down).

the stiffness matrix and the modes of finite element model of the nominal plate. Let $\left[M^{\text {mod }}\right],\left[K^{\text {mod }}\right]$ and $\left[\phi^{\text {mod }}\right]$ be the
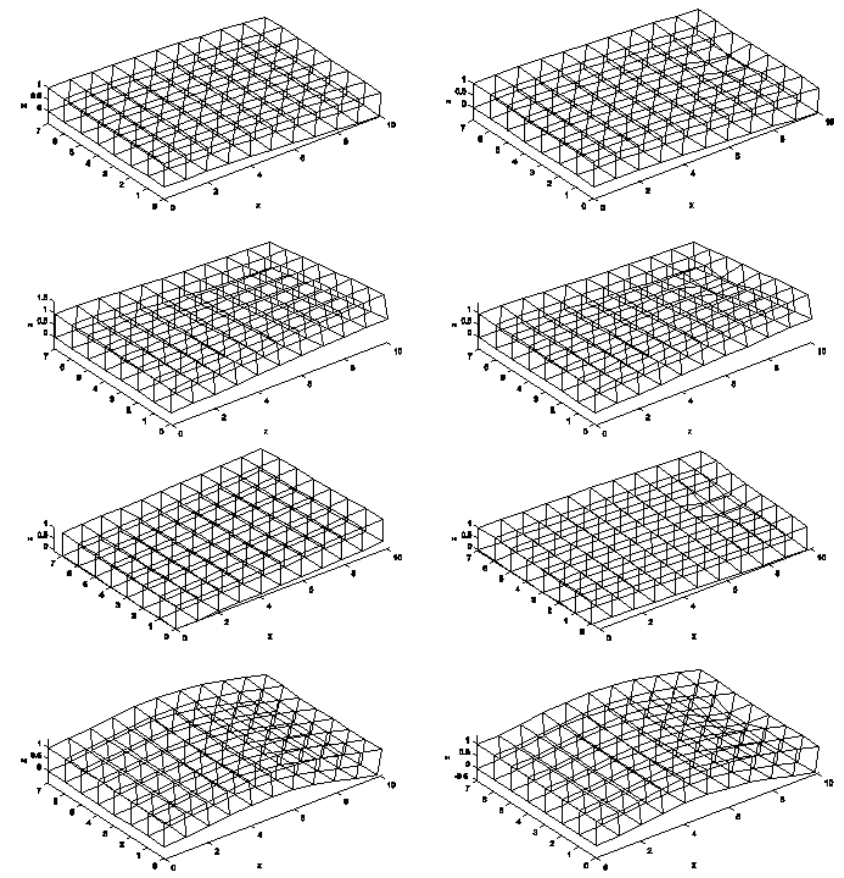

Fig. 13. 4 first modes of the nominal plate (on left) and of the geometrically modified plate (on right). From up to down: mode 1 , mode 2, mode 3, mode 4.

mass matrix, the stiffness matrix and the modes of finite element model of the geometrically modified plate. The eigenfrequencies of the modified plate are then computed by two different ways:

1. Directly by solving the eigenvalue problem defined by: Find $\left(\lambda_{d}^{\text {mod }}, \phi_{d}^{\text {mod }}\right)$ so that

$$
\left(\left[K^{\text {mod }}\right]-\lambda_{d}^{\text {mod }}\left[M^{\text {mod }}\right]\right) \phi_{d}^{\text {mod }}=0
$$

2. By reducing the mass and stiffness matrices of the geometrically modified plate using a basis of modes of the nominal plate and by solving the eigenvalue problem defined by: Find $\left(\lambda_{r}^{\text {mod }}, \phi_{r}^{\text {mod }}\right)$ so that

$$
\left(\left[K_{\text {red }}^{\text {mod }}\right]-\lambda_{r}^{\text {mod }}\left[M_{\text {red }}^{\text {mod }}\right]\right) \phi_{r}^{\text {mod }}=0,
$$

where

$$
\left[K_{\text {red }}^{\text {mod }}\right]=\left[\phi^{\text {nom }}\right]^{T}\left[K^{\text {mod }}\right]\left[\phi^{\text {nom }}\right]
$$

$$
\left[M_{\text {red }}^{\text {mod }}\right]=\left[\phi^{\text {nom }}\right]^{T}\left[M^{\text {mod }}\right]\left[\phi^{\text {nom }}\right]
$$

When we try to obtain the natural frequencies of the modified plate by using a reduced model obtained by projecting the modified plate mass and stiffness matrices on the nominal plate modes, and when we compare them to the natural 
frequencies directly computed, high error levels appear in the estimation of the natural frequencies. Table 2 shows the high error levels obtained for the 4 first modes by using a basis containing the 50 first modes. In fact, for approximating the natural frequency of mode 2 , the error level exceeds $35 \%$. Even by proceeding to a convergence analysis of the

\begin{tabular}{|c|c|c|c|}
\hline & $\begin{array}{c}\text { Direct solving } \\
\text { frequency }(\mathrm{Hz})\end{array}$ & $\begin{array}{c}\text { Reduced model } \\
\text { frequency }(\mathrm{Hz})\end{array}$ & $\begin{array}{c}\text { Frequency } \\
\text { error (\%) }\end{array}$ \\
\hline Mode 1 & 10,79 & 13,70 & 27,01 \\
\hline Mode 2 & 27,56 & 37,47 & 35,92 \\
\hline Mode 3 & 46,44 & 46,45 & 0,006 \\
\hline Mode 4 & 63,89 & 70,17 & 9,82 \\
\hline
\end{tabular}

Table 2. Natural frequencies and frequency error levels obtained between the reduced model and the direct computation.

obtained frequencies, with respect to the modal projection basis dimension, we see that the convergence speed is low. So to get accurate results with this kind of domain incompatibilities, by only using modes of the nominal plate, one could have a reduced-order model of great size.

\section{Acknowledgments}

Turbomeca Company is gratefully acknowledged for the permission to publish this work. The authors wish to thank reviewers for their interesting comments.

\section{References}

[1] Whitehead, D., 1966. "Effects of Mistuning on the Vibration of Turbomachine Blades Induced by Wakes". Journal of Mechanical Engineering Science, 8(1), pp. 15-21.

[2] Dye, R., and Henry, T., 1969. "Vibration Amplitudes of Compressor Blades Resulting from Scatter in Blade Natural Frequencies". ASME Journal of Engineering for Power, 91(3), pp. 182-187.

[3] Capiez-Lernout, E., and Soize, C., 2004. "Nonparametric Modeling of Random Uncertainties for Dynamic Response of Mistuned Bladed-disks". ASME Journal of Engineering for Gas Turbines and Power, 126(3), pp. 610-618.

[4] Ewins, D., 1969. "The Effects of Detuning upon the Forced Vibrations of Bladed Disks". Journal of Sound and Vibration, 9(1), pp. 65-69.

[5] Choi, B.-K., Lentz, J., Rivas-Guerra, A., and Mignolet, M., 2003. "Optimization of Intentional Mistuning Patterns for the Reduction of the Forced Response Effects of Unintentional Mistuning: Formulation and Assessment". ASME Journal of Engineering for Gas Turbines and Power, 125, pp. 131-140.
[6] Castanier, M., Ottarson, G., , and Pierre, C., 1997. "Reduced Order Modeling Technique for Mistuned Bladed Disks". ASME Journal of Vibration and Acoustics, 119(3), pp. 439-447.

[7] Castanier, M., and Pierre, C., 2002. "Using Intentional Mistuning in the Design of Turbomachinery rotors". AIAA Journal, 40(10), pp. 2077-2086.

[8] Mignolet, M., Hu, W., and Jadic, I., 2000. "On the Forced Response of Harmonically and Partially Mistuned Bladed Disks. part 1: Harmonic Mistuning, part 2: Partial Mistuning and Applications". International Journal of Rotating Machinery, 6(1), pp. 29-56.

[9] Thomas, D., 1979. "Dynamics of Rotationally Periodic Structures”. International Journal for Numerical Methods in Engineering, 14(1), pp. 81-102.

[10] Jacquet-Richardet, G., Ferraris, G., and RIeutord, P., 1996. "Frequencies and modes of rotating flexible bladed disc-shaft assemblies: A global cyclic symmetry approach". Journal of Sound and Vibration, 191(5), pp. 901-915.

[11] Williams, F., Kennedy, D., Wu, G., and Zhou, J., 1996. "Exact computation of natural frequencies of repetitive structures". Structural Engineering and Mechanics, 4(5), pp. 553-568.

[12] Ohayon, R., and Soize, C., 1998. Structural acoustics and vibration. Academic press.

[13] Bladh, R., Castanier, M., and Pierre, C., 1999. "Reduced order modeling and vibration analysis of mistuned bladed disk assemblies with shrouds". ASME Journal of Engineering for Gas Turbines and Power, 121(3), pp. 515-522.

[14] Bladh, R., Castanier, M., and Pierre, C., 2001. "Component-mode-based reduced order modeling techniques for mistuned bladed disks - part i: Theoretical models". ASME Journal of Engineering for Gas Turbines and Power, 123(1), pp. 89-99.

[15] Yang, M.-T., and Griffin, J., 2001. "A Reduced-Order Model of Mistuning using a Subset of Nominal System Modes". ASME Journal of Engineering for Gas Turbines and Power, 123(3), pp. 893-900.

[16] Feiner, D., and Griffin, J., 2002. "A fundamental model of mistuning for a single family of modes". Journal of Turbomachinery, 124, pp. 597-605.

[17] Feiner, D., and Griffin, J., 2002. "Mistuning identification of bladed disks using fundamental mistuning model. part 1: Theory, part 2: Application”. Journal of Turbomachinery, 126, pp. 150-165.

[18] Lim, S.-H., Castanier, M., and Pierre, C., 2004. "Vibration modeling of bladed disks subject to geometric mistuning and design changes". In Proceedings of the 45-th AIAA/ASME/ASCE/AHS/ASC Structures, Structural Dynamics and Material Comference, paper 2004-1686, Palm Springs, California, USA.

[19] Martel, C., Corral, R., and Llorens, J., 2008. "Stability increase of aerodynamically unstable rotor using intentional mistuning". In Proceedings of the ASME TURBO EXPO 2008.

[20] Martel, C., and Corral, R., 2008. “Asymptotic descrip- 
tion of maximum mistuning amplification of bladed disk forced response". In Proceedings of the ASME TURBO EXPO 2008.

[21] Sinha, A., 2009. "Reduced-order model of a bladed rotor with geometric mistuning". Journal of Turmomachinery - Transaction of the ASME, 131, pp. 031007.1 - 031007.7.

[22] Ganine, V., Legrand, M., Pierre, C., and Michalska, H., 2008. "A reduction technique for mistuned bladed disks with superposition of large geometric mistuning and small model uncertainties". In Proceedings of the 12-th International Symposium on Transport Phenomena and Dynamics of Rotating Machinery, paper 200820158, Honolulu, Hawaii, USA.

[23] Ohayon, R., Sampaio, R., and Soize, C., 1997. "Dynamic Substructuring of Damped Structures using the Singular Value Decomposition". ASME Journal of Applied Mechanics, 64(2), pp. 292-298. 